\title{
2D and 3D in vitro assays to quantify the invasive behavior of glioblastoma stem cells in response to SDF-1 $\alpha$
}

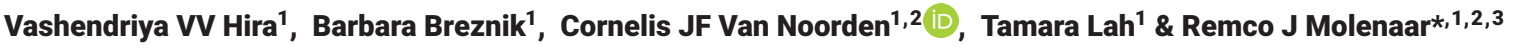 \\ ${ }^{1}$ Department of Genetic Toxicology \& Cancer Biology, National Institute of Biology, Ljubljana, Slovenia; ${ }^{2}$ Department of Medical Biology, Cancer Center Amsterdam, \\ Amsterdam UMC at The Academic Medical Center, Amsterdam, The Netherlands; ${ }^{3}$ Department of Medical Oncology, Cancer Center Amsterdam, Amsterdam UMC at \\ The Academic Medical Center, Amsterdam, The Netherlands; *Author for correspondence: r.j.molenaar@amsterdamumc.nl
}

BioTechniques 69: 339-346 (November 2020) 10.2144/btn-2020-0046

First draft submitted: 14 April 2020; Accepted for publication: 27 July 2020; Published online: 1 September 2020

\section{ABSTRACT}

Invasion is a hallmark of cancer and therefore in vitro invasion assays are important tools in cancer research. We aimed to describe in vitro $2 \mathrm{D}$ transwell assays and $3 \mathrm{D}$ spheroid assays to quantitatively determine the invasive behavior of glioblastoma stem cells in response to the chemoattractant SDF-1 $\alpha$. Matrigel was used as a matrix in both assays. We demonstrated quantitatively that SDF-1 $1 \alpha$ increased invasive behavior of glioblastoma stem cells in both assays. We conclude that the 2D transwell invasion assay is easy to perform, fast and less complex whereas the more time-consuming 3D spheroid invasion assay is physiologically closer to the in vivo situation.

\section{METHOD SUMMARY}

We describe 2D transwell invasion assays and 3D spheroid invasion assays for the investigation of effects of the chemoattractant SDF-1 $\alpha$ on human glioblastoma stem cells in vitro in a quantitative manner using image analysis. In both in vitro invasion assays, Matrigel was used as the matrix that glioblastoma stem cells invade. The 2D assay is easy to perform, fast and less complex whereas the 3D assay models the in vivo situation more closely.

\section{KEYWORDS:}

2D transwell invasion assay $\bullet$ 3D spheroid invasion assay $\bullet$ cancer cell $\bullet$ cellular invasion $\bullet$ glioblastoma stem cells

Invasive behavior is a hallmark of cancer and a major reason why the disease is hard to eradicate, causing poor patient prognosis [1-3]. Invasive cancer cells migrate through the extracellular matrix (ECM), enabled by the secretion of proteases to degrade the ECM and alterations in the cytoskeleton [4-6].

Multiple in vitro assays and models are available to study cellular migration and invasion, such as the scratch assay, 3D bioscaffolds and microfluidic co-cultures [3,7-11]. Frequently used invasion assays are the transwell invasion assay (2D invasion assay) [3,8,12,13] and the spheroid invasion assay (3D invasion assay) [5,12,14-16]. The latter two types of invasion assays are discussed in this article. Both types of in vitro invasion assays are relatively easy to perform, fast, reproducible and cheap. Although the 3D invasion assay is more complex than the 2D invasion assay, it is physiologically more relevant because it represents the in vivo situation in solid tumors more accurately. In 3D invasion assays, cancer cells not only invade through a matrix, but are also affected by other cancer cells in their direct surroundings. Cancer cells need to change and modify their surrounding environment in order to invade, which is also the case in the in vitro invasion assays [1,17]. In vitro invasion assays are generally preferred over in vivo invasion experiments using fluorescently-labeled cells and intravital imaging in animal models because of ethical concerns and the high costs of animal models. Furthermore, the in vitro invasion assays are more controllable, more flexible and easier to modify $[1,18,19]$.

In this article, we describe the in vitro 2D transwell invasion assay and the 3D spheroid invasion assay in detail with the use of a brain tumor patient-derived glioblastoma stem cell (GSC) line. GSCs have been used instead of differentiated glioblastoma cells, because they have a higher invasive potential than differentiated glioblastoma cells $[20,21]$. The presence of cell surface protein L1CAM and the intracellular PI3K/AKT and Notch signaling pathways are involved in the highly elevated invasive potential of GSCs compared with differentiated glioblastoma cells [21,22]. Therefore, GSCs are a suitable cell type to demonstrate cellular invasion. GSCs as well as differentiated glioblastoma cells express CXCR4, a specific receptor for the chemoattractant SDF-1 $\alpha$, which we found to be highly expressed in hypoxic peri-arteriolar GSC niches in glioblastoma patient samples where GSCs are maintained in the tumors and protected from chemotherapy and radiotherapy because of their quiescence [23-25]. Interactions between SDF-1 $\alpha$ and CXCR4 have been reported 


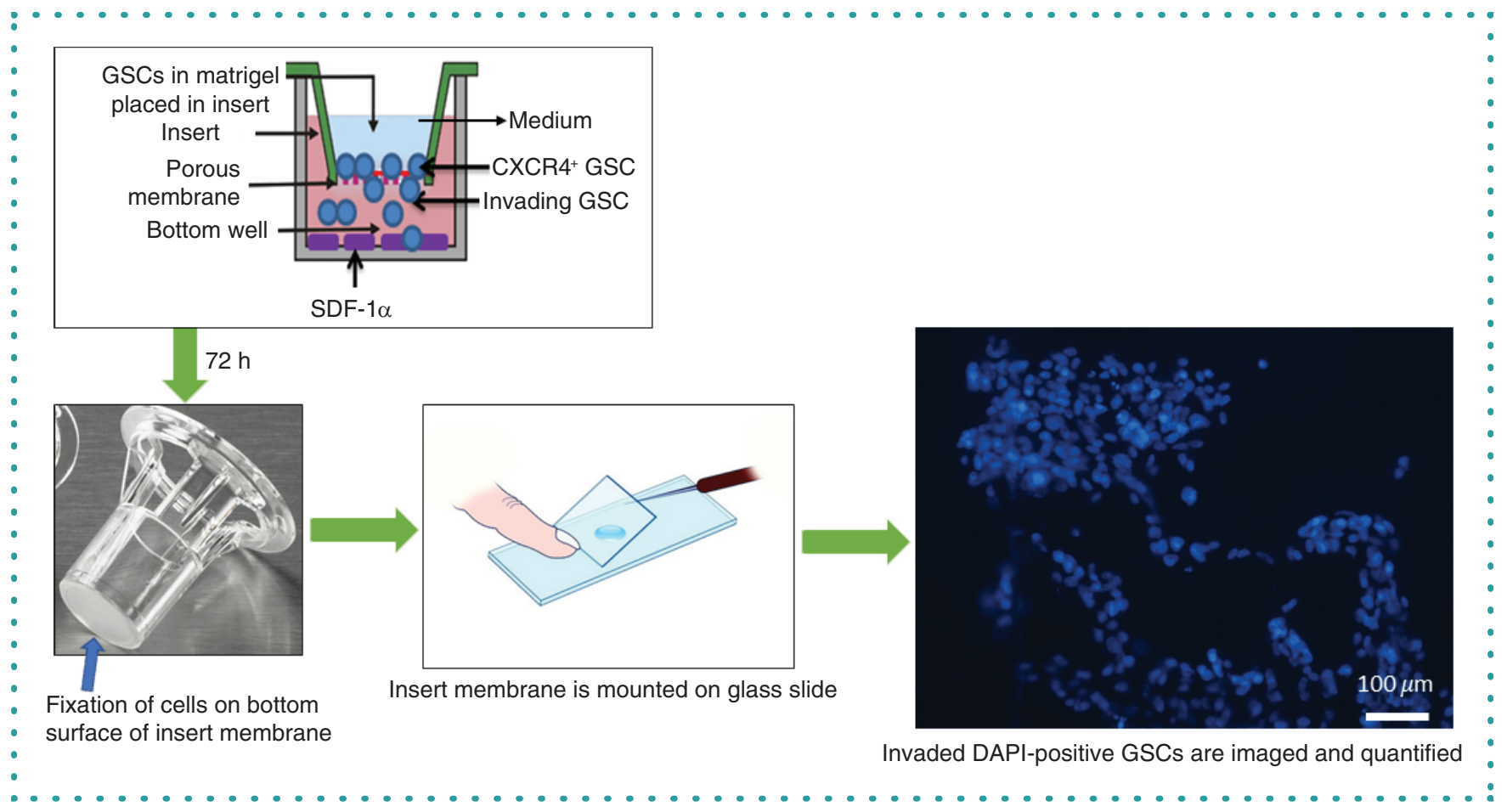

Figure 1. Schematic overview of the 2D transwell invasion assay. CXCR4-positive GSCs in Matrigel-containing medium are placed in the top wells (inserts) in the presence or absence of chemoattractant SDF-1 $\alpha$ in the medium in bottom wells. Invaded GSCs accumulate at the bottom surface of the insert membrane (blue arrow). After $72 \mathrm{~h}$, cells on the bottom surface of insert membranes are fixed and the membrane is cut out of the insert. The membrane is mounted on a microscopic slide, followed by imaging and quantification of DAPI-positive GSCs using image analysis. Scale bar $=100 \mu \mathrm{m}$. DAPI: 4',6-diamidino-2-phenylindole; GSC: Glioblastoma stem cell.

to be involved in cellular invasive behavior in glioblastoma [26-29] and the SDF-1 $\alpha$-CXCR4 axis enables invasion of CXCR4-positive glioblastoma cells into SDF-1 $\alpha$-rich niches and transformation into GSCs [23-25].

In the 2D and 3D invasion assays, Matrigel is used as a matrix. In this study we aimed to quantitatively determine the invasive cellular behavior of CXCR4-expressing GSCs in the presence and absence of SDF- $1 \alpha$. In addition, we aimed to assess the advantages and disadvantages of both types of in vitro invasion assays.

\section{Materials \& methods}

Cell culture

NCH421k GSCs [30,31] were a generous gift from Christel Herold-Mende (Heidelberg University, Heidelberg, Germany) and were cultured as non-adherent 3D spheroids in Neurobasal ${ }^{\top \mathrm{M}}$ medium (Gibco, Life Technologies, CA, USA) containing 1\% penicillin/streptomycin (Sigma, MO, USA), 1\% L-glutamine (Sigma), 2\% B27 (Gibco), 0.08\% bFGF (Gibco), 0.01\% EGF (Gibco) and $0.01 \%$ heparin (Sigma) at $37^{\circ} \mathrm{C}$ in a $5 \%$ $\mathrm{CO}_{2}$ incubator.

\section{D transwell invasion assay}

The method of the transwell invasion assay is described step by step, to be performed chronologically to reproduce the experiment. $\mathrm{NCH} 421 \mathrm{k}$ GSCs growing as 3D spheroids were mechanically resuspended to obtain single cells. Transwell invasion assays as shown in Figure 1 were performed using inserts with 8.0- $\mu$ m pores (Corning Life Sciences, NY, USA). NCH421k GSCs (80,000 cells/insert) were used that express CXCR4 as described elsewhere [12,24]. Cells were mixed in $0.5 \mathrm{mg} / \mathrm{ml}$ Matrigel (Corning) and plated in inserts in a total volume of $50 \mu \mathrm{l}$, and the inserts were then placed in 24-well plates (Corning). After incubation for $30 \mathrm{~min}$ at $37^{\circ} \mathrm{C}$ in a $5 \% \mathrm{CO}_{2}$ incubator, $50 \mu \mathrm{l}$ complete Neurobasal medium was added to the inserts to obtain a total volume of $100 \mu \mathrm{l}$. For the experimental condition, $600 \mu \mathrm{l}$ complete Neurobasal medium containing $10 \mathrm{ng} / \mathrm{ml}$ SDF-1 $\alpha$ (catalog \#: 300-28A; Peprotech, NJ, USA) was added to the bottom wells. As a control, complete Neurobasal medium without SDF-1 $\alpha$ was added to the bottom wells. After $72 \mathrm{~h}$, invaded cells that accumulated on the bottom surface of the insert membranes were fixed with $4 \%$ paraformaldehyde (Merck, Darmstadt, Germany) for 20 min at room temperature, washed with $1 \times$ phosphate-buffered saline (PBS; Gibco) for $10 \mathrm{~min}$ and the cell nuclei were stained with the DNA dye 4',6diamidino-2-phenylindole (DAPI; Sigma) for $5 \mathrm{~min}$, followed by a washing step with $1 \times$ PBS for $10 \mathrm{~min}$. After removing the cells and Matrigel out of the inserts with cotton swabs, the membranes were cut out from the inserts with a scalpel and mounted on microscopic slides using Prolong Gold mounting medium (Life Technologies, CA, USA) for quantification of invaded DAPI-positive cells (Figure 1). 


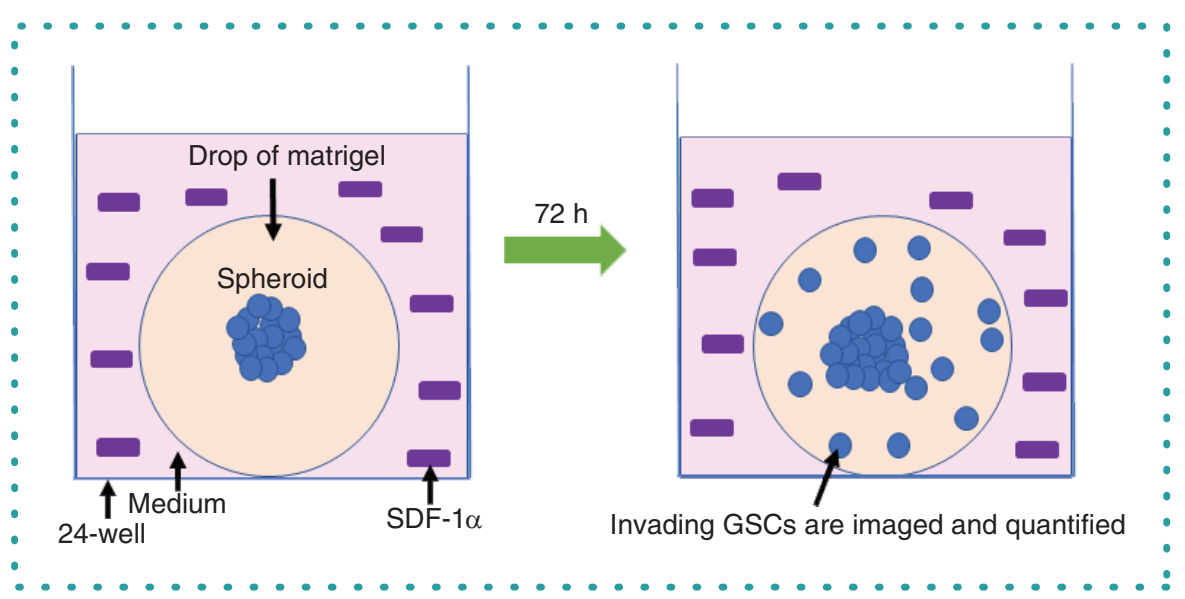

Figure 2. Schematic overview of the 3D spheroid invasion assay. After spheroid preparation in U-bottomed 96 wells, single GSC spheroids are transferred into 24-wells and embedded in a drop of Matrigel. The spheroids are covered with Neurobasal medium in the presence or absence of SDF- $1 \alpha$. After $72 \mathrm{~h}$ of incubation at $37^{\circ} \mathrm{C}$, images of the spheroids are captured and the GSCs that invade from the spheroid into the Matrigel-containing medium are quantified using image analysis.

GSC: Glioblastoma stem cell.

Images of the membranes were captured using a Nikon Eclipse Ti-E fluorescence inverted microscope and the NIS-Elements AR 4.13.04 software (Nikon, Tokyo, Japan). The total number of cells in ten individual fields per condition was counted using image analysis and the Image J software [32,33]. Three experiments were performed, each in triplicate.

Image analysis was performed using ImageJ software and the following tools: $\rightarrow$ Analyze $\rightarrow$ Cell counter. Cells were counted by clicking on all DAPI-positive nuclei (Figure 1).

\section{Ki67 expression to determine cell proliferation in transwell invasion assays}

In order to determine whether invasion of GSCs is affected by cell proliferation during the transwell invasion assays, transmembranes were stained using the proliferation biomarker Ki67. After fixation of the cells with $4 \%$ paraformaldehyde, cell membranes were permeabilized using PBS containing $1 \%$ bovine serum albumin and $0.1 \%$ Triton- $X$ for $30 \mathrm{~min}$ at room temperature. Afterward, transmembranes were incubated with anti-Ki67 antibodies conjugated with fluorescein isothiocyanate (Miltenyi Biotec, CA, USA; catalog number: 130-117691) in a dilution of 1:50 in PBS at room temperature for $1 \mathrm{~h}$. After one washing step with PBS, transmembranes were stained with DAPI for $5 \mathrm{~min}$, followed by a washing step with $1 \times$ PBS for $10 \mathrm{~min}$. Transmembranes were cut out of the inserts and mounted on microscopic slides using Prolong Gold mounting medium for quantification of invaded DAPI-positive and Ki67-positive cells. Images of the transmembranes were taken using a Nikon Eclipse Ti-E inverted microscope and the NIS-Elements AR 4.13.04 software. The total number of cells in ten individual fields per transmembrane was counted using image analysis and Image $\mathrm{J}$ software $[32,33]$. Three experiments were performed, each in triplicate.

\section{Three-dimensional spheroid invasion assay}

The method of the spheroid invasion assay is described step by step, to be performed chronologically to reproduce the experiment. $\mathrm{NCH} 421 \mathrm{k}$ GSCs were seeded in complete Neurobasal medium containing $4 \%$ methylcellulose in U-bottomed 96 -well plates with 3000 cells/well (BD Biosciences, CA, USA). The $4 \%$ methylcellulose was prepared as follows: $6 \mathrm{~g}$ of pure methylcellulose powder (Sigma, catalog \# m-0512) was autoclaved and dissolved in $250 \mathrm{ml}$ pre-heated complete Neurobasal medium $\left(60^{\circ} \mathrm{C}\right)$ for 20 min using a magnetic stirrer. Then $250 \mathrm{ml}$ complete Neurobasal medium (room temperature) was added, to a final volume of $500 \mathrm{ml}$, and this solution was mixed for $2 \mathrm{~h}$ at $4{ }^{\circ} \mathrm{C}$. The final stock solution was aliquoted and cleared by centrifugation $(5000 \times g, 2 \mathrm{~h}$, room temperature). Only the clear highly viscous supernatant - approximately $90-95 \%$ of the stock solution - was used for the spheroid invasion assay. The cells in U-bottomed 96 -well plates were centrifuged at $850 \times \mathrm{g}$ and $31^{\circ} \mathrm{C}$ for $90 \mathrm{~min}$ and incubated at $37^{\circ} \mathrm{C}$ in a $5 \% \mathrm{CO}_{2}$ incubator for $72 \mathrm{~h}$ to form a single spheroid of similar size in each well. Spheroids were transferred into 24-well plates (Corning) and embedded in $6.0 \mathrm{mg} / \mathrm{ml}$ Matrigel (Figure 2). After $30 \mathrm{~min}$ of incubation at $37^{\circ} \mathrm{C}$ in a $5 \% \mathrm{CO}_{2}$ incubator, spheroids were covered with complete Neurobasal medium containing SDF-1 $\alpha(10 \mathrm{ng} / \mathrm{ml})$. As a control, complete Neurobasal medium without SDF-1 $\alpha$ was used to cover the spheroids. Microscopical images were captured after $72 \mathrm{~h}$ using a Nikon Eclipse Ti inverted fluorescence microscope and NIS-Elements AR 4.13.04 software. The number of GSCs that invaded from the spheroids into the Matrigel was determined using image analysis and the NIS-Elements software (Figure 2) [32,33]. GSC spheroids with a minimum diameter of $40 \mu \mathrm{m}$ were considered as spheroids [11]. Three experiments were performed, each in triplicate. 


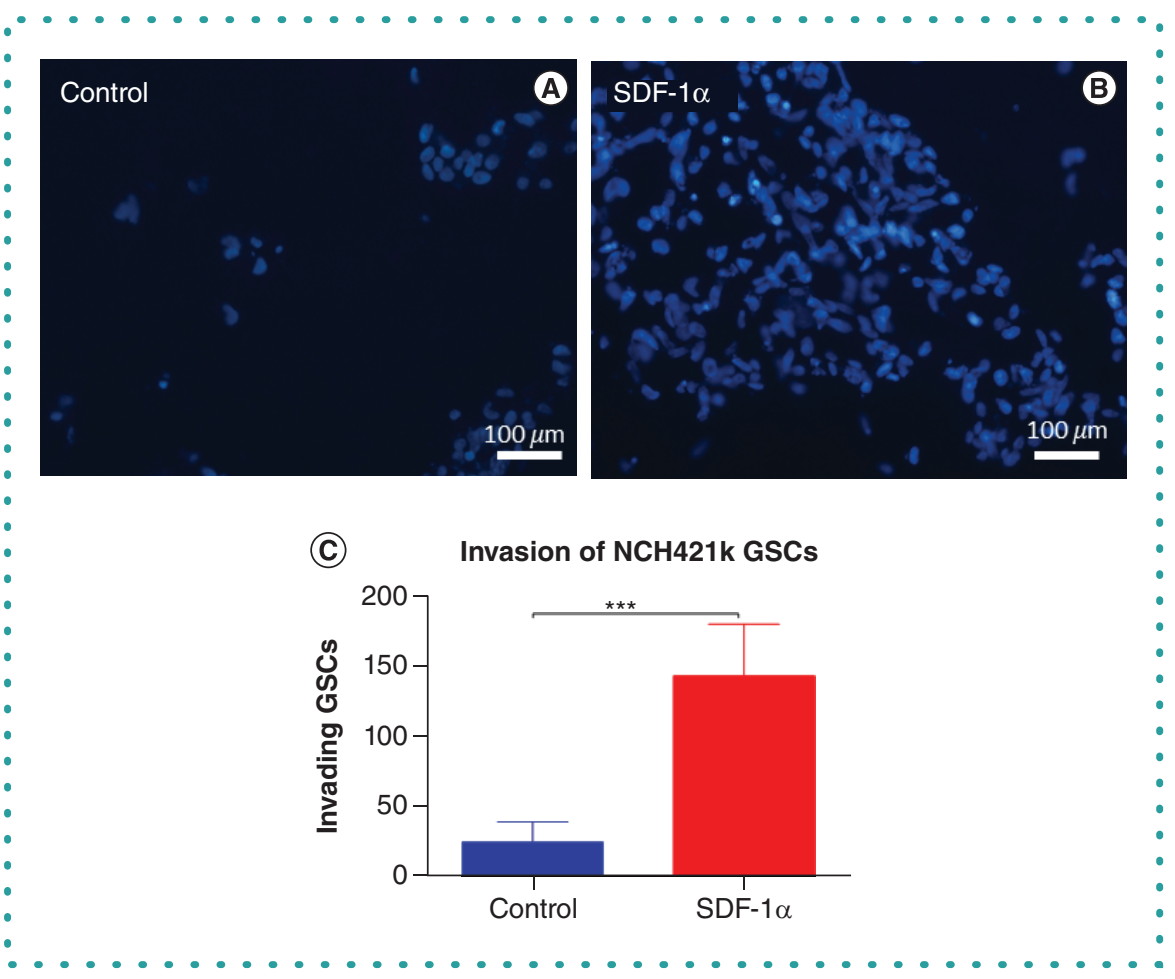

Figure 3. 2D transwell invasion data of CXCR4-positive NCH421k glioblastoma stem cells. GSCs that accumulated on the bottom surface of the insert membranes were imaged at $72 \mathrm{~h}$. DAPI staining was used to show cell nuclei. In the presence of the chemoattractant SDF- $1 \alpha$ in bottom wells, a significant increase in the average number of invading GSCs was observed as compared with the control condition without SDF-1 $\alpha$. Bars represent the mean number ( \pm standard error of the mean) of invading NCH421k GSCs after $72 \mathrm{~h}$ of incubation in control conditions (empty medium) or in medium containing $10 \mathrm{ng} / \mathrm{ml} \mathrm{SDF-1} \alpha$.

Scale bar $=100 \mu \mathrm{m} . * \star \star \mathrm{p}<0.001$.

DAPI: 4',6-diamidino-2-phenylindole; GSC: Glioblastoma stem cell.

\section{Statistical analyses}

Data was processed in Excel 2013 (Microsoft, WA, USA) and GraphPad Prism 6 (GraphPad, CA, USA) for statistical analyses using oneway analysis of variance; $p$-values lower than 0.05 were considered to indicate significant differences.

\section{Results \& discussion}

\section{CXCR4-positive GSCs are attracted to SDF-1 $\alpha$ in 2D transwell invasion assays}

Transwell invasion assays were performed using CXCR4-positive NCH421k GSCs in Matrigel-containing medium in inserts in the presence or absence of the chemoattractant SDF-1 $\alpha$ in the bottom wells. Imaging of DAPI-positive GSCs on the bottom surface of insert membranes was performed at $72 \mathrm{~h}$ (Figure 3). Our data demonstrate a significant (sevenfold) increase in the average number of invading GSCs in the presence of SDF-1 $\alpha$ (Figure 3B \& C) as compared with the control condition, where empty medium was added to the bottom wells (Figure $3 \mathrm{~A} \& \mathrm{C}$ ). Only cells that had accumulated on the bottom surface of insert membranes were included as no cells were found in the bottom wells.

\section{CXCR4-positive GSCs invade into Matrigel in the presence of SDF-1 $\alpha$ in 3D spheroid invasion assays}

In the 3D invasion assay, spheroids of CXCR4-positive NCH421k GSCs were embedded in Matrigel and covered with medium with or without SDF-1 $\alpha$ (Figure 4). At $72 \mathrm{~h}$, imaging was performed and the number of GSCs that invaded out of the spheroid was quantified. Our data demonstrate that the presence of SDF-1 $\alpha$ resulted in a 1.8-fold higher number of GSCs invading from the spheroid into the Matrigel-containing medium (Figure 4B \& C), as compared with the control condition (Figure 4A \& C).

\section{Proliferation of GSCs is not affected during invasion assays}

To determine whether or not proliferation of GSCs is affected during invasion assays, antibodies against the proliferation biomarker Ki67 were used to detect proliferating cells on the membrane of inserts in $2 \mathrm{D}$ transwell invasion assays (Figure $5 \mathrm{~A}$ ). Our data demonstrate that proliferation of NCH421K GSCs was not affected; the percentage of Ki67-positive GSCs did not differ between control conditions and the wells where SDF- $1 \alpha$ was added (Figure 5A). In the 3D spheroid invasion assays, we assessed that cells that invaded out of spheroids were always single cells and not clusters of cells (Figure 5B), confirming that GSC proliferation did not take place during invasion. 


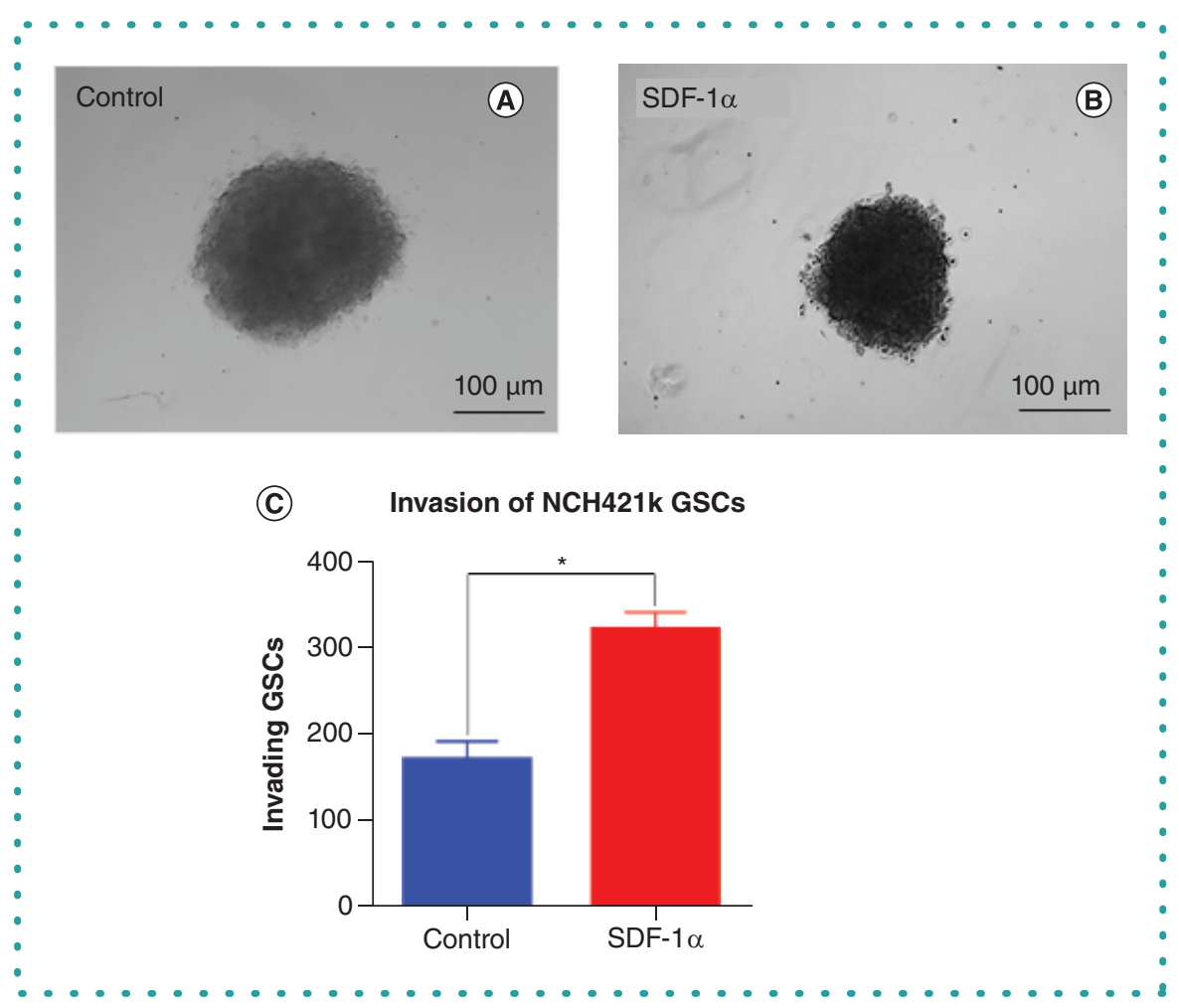

Figure 4. 3D spheroid invasion data of CXCR4-positive NCH421k glioblastoma stem cells. GSC spheroids were embedded in a drop of Matrigel that was covered with empty medium (A) or medium containing SDF-1 $\alpha$ (B). (C) Higher numbers of GSCs invaded from the spheroid in the presence of SDF-1 $\alpha$ as compared with the control condition. Bars represent the mean number ( \pm standard error of the mean) of invading NCH421k GSCs after $72 \mathrm{~h}$ from the spheroids into the Matrigel in control conditions (empty medium) or in medium containing $10 \mathrm{ng} / \mathrm{ml} \mathrm{SDF-1 \alpha}$. Scale bar $=100 \mu \mathrm{m}$. ${ }^{\star} \mathrm{p}<0.05$.

GSC: Glioblastoma stem cell.

Taken together, our data indicate that SDF-1 $\alpha$ attracts CXCR4-positive GSCs in 2D transwell invasion assays and induces invasion of CXCR4-positive GSCs into the Matrigel in 3D spheroid invasion assays. Proliferation is not affected during invasion of GSCs in either type of in vitro invasion assay.

In this article, we describe two quantitative assays for the evaluation of GSC invasion in vitro - the 2D transwell invasion assay and a 3D spheroid invasion assay - and assess the advantages and disadvantages of each.

Our data demonstrate that both in vitro invasion assays are feasible experimental methods to investigate the cellular invasive behavior of cancers with invasive properties, such as glioblastoma. As an example, we demonstrate that CXCR4-positive GSCs are attracted toward SDF-1 $\alpha$ in bottom wells in the 2D transwell invasion assays (Figure 3 ) and in Matrigel-containing medium around spheroids in the 3D spheroid invasion assay (Figure 4). GSCs invaded out of spheroids as single cells (Figures 4 \& 5B), as reported in our previous study [12]. We also demonstrate that cell proliferation is not affected in either type of in vitro invasion assay (Figure 5). This is in line with studies that reported the 'go or grow' hypothesis, which states that cancer cells either invade or proliferate, because cellular invasion and proliferation do not occur simultaneously [34,35].

The major advantages of the 2D transwell and 3D spheroid invasion assays for the in vitro evaluation of GSC invasion are that they are technically easy to perform, relatively cheap and reproducible [3]. They can also be applied to other types of cancer (stem) cells and are now also used for drug testing, to determine whether drugs can inhibit the invasive behavior of cancer (stem) cells and their spheroid formation ability [36-41]. Chemotaxis, such as attraction between CXCR4 and SDF-1 $\alpha$, can be studied in an accurate manner using both assays [12]. In both assays, the presence of SDF-1 $\alpha$ clearly results in increased invasive behavior of CXCR4-positive GSCs (Figures 3 \& 4). Another advantage of $2 \mathrm{D}$ transwell and $3 \mathrm{D}$ spheroid invasion assays is that qualitative data are obtained by imaging (differences in numbers of invading GSCs can be clearly observed microscopically) and quantitative data are obtained using image analysis, which is relatively easy to perform and accurate at the same time. Alternatively, a quantitative microscopic analysis has been described for the $3 \mathrm{D}$ spheroid assay in which the distance of invading cells from the spheroid or invasive area is determined [15,42].

Both in vitro invasion assays can be performed in more complicated experimental settings by using multiple cell types - for example, CXCR4-positive GSCs in the inserts and SDF-1 $\alpha$-producing cells in the bottom wells, such as mesenchymal stem cells (MSCs) that are known to infiltrate glioblastoma tumors and are major SDF-1 $\alpha$ producers [24]. Compared with experiments with exogenous SDF-1 $\alpha$ 


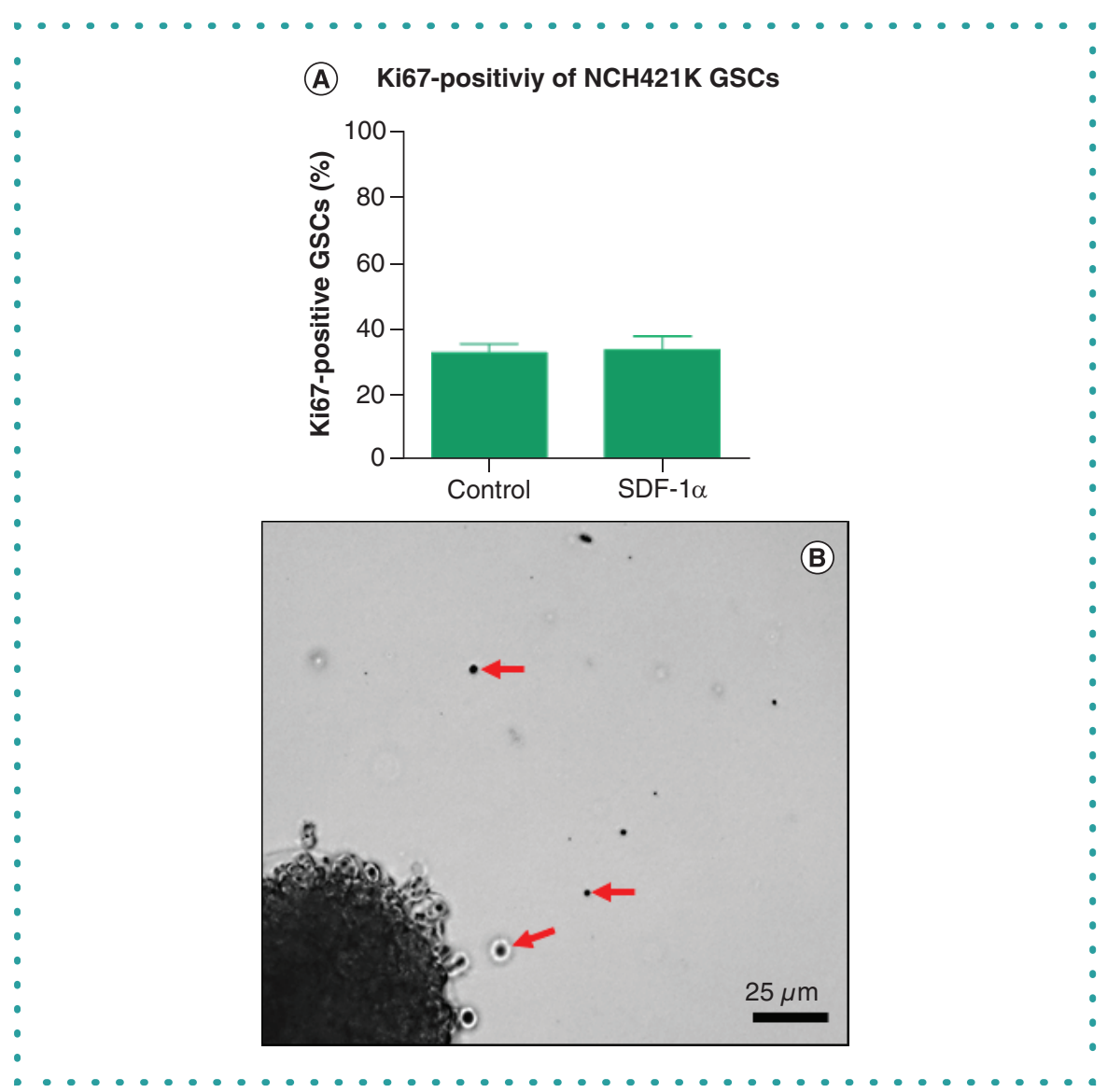

Figure 5. Proliferation of $\mathrm{NCH} 421 \mathrm{k}$ glioblastoma stem cells was not affected during invasion assays. In the 2D transwell invasion assay, Ki67 staining was performed on GSCs that accumulated on the bottom surface of insert membranes to detect proliferating GSCs in transwell invasion assays. Imaging was performed after $72 \mathrm{~h}$. (A) No differences were found between the percentages of proliferating Ki67-positive GSCs in control conditions and in the presence of SDF-1 $\alpha$ in bottom wells. (B) NCH421k GSCs that invaded out of the spheroid in 3D spheroid invasion assays were single cells, which confirms that invading GSCs are not proliferating. Bars represent the mean number ( \pm standard error of the mean) of invading NCH421k GSCs in the 2D transwell invasion assay after $72 \mathrm{~h}$ of incubation in control conditions (empty medium) or in medium containing $10 \mathrm{ng} / \mathrm{ml} \mathrm{SDF}-1 \alpha$. Scale bar $=25 \mu \mathrm{m}$. GSC: Glioblastoma stem cell.

present in the bottom wells, such experiments are both more biologically relevant (because SDF-1 $\alpha$ is produced by MSCs in GSC niches in vivo) and cheaper (fewer reagents are needed because MSCs produce SDF- $1 \alpha$ ). However, it should be noted that MSCs produce a plethora of chemokines, not only SDF-1 $\alpha[12,43]$. Thus, to determine whether the obtained effect was caused by MSC-secreted SDF$1 \alpha$, control experiments need to be performed using selective CXCR4 inhibitors like plerixafor [44]. In the 3D spheroid invasion assays, spheroid co-cultures of different fluorescently-labeled cell types can also be used to study cancer cell invasion into the 3D matrix [14]. A disadvantage of these two invasion methodologies is that the tumor microenvironment is not accurately reproduced $[3,8]$. More advanced and expensive in vitro 3D invasion methods are now available, such as 3D microfluidic co-culture systems that allow the construction of a 3D tumor microenvironment, real-time cell tracking, evaluation of interactions between different cell types, mimicking of blood flow and studies of phenomena such as cell proliferation and invasion $[3,8,11,45-47]$. The most true-to-nature systems are the 3D tumor organoids, in which the tumor complexity (i.e., tumor structures including blood vessels, stroma, ECM) is intact and tumor heterogeneity is present. Organoids can be used for drug testing as well for the investigation of phenomena such as cellular invasive behavior [48-55]. In future research, we aim to introduce 3D tumor organoids as a model to study glioblastoma invasion and interactions between glioblastoma cells/GSCs with other cell types in the tumor microenvironment, but both 2D transwell invasion assay and 3D spheroid invasion assay will continue to be used as well because of their own advantages. The $2 \mathrm{D}$ transwell invasion assay is a faster method and is less complex than the 3D spheroid invasion assay. However, it is more expensive than the 3D invasion assay because of the costs of inserts. Therefore, we suggest that the $2 \mathrm{D}$ transwell assay is applied first to determine whether the hypothesized chemoattractant-receptor interactions are involved in the process of cellular invasion. Afterward, cellular invasion can be studied using the more time-consuming 3D spheroid invasion assays that are more complex and closer to the in vivo situation. A limitation of the present study is that both in vitro invasion assays have been performed with the use of a single GSC line only. The application of this protocol to additional patient-derived GSC lines in the future may enable the optimization of this methodology for individual cell lines. 
Besides the SDF-1 $\alpha$-CXCR4 axis, various other signaling axes are involved in the invasive behavior of GSCs, such as the intracellular PI3K/AKT and Notch signaling pathways [21,22]. In addition, proteases such as matrix metalloproteinases (e.g., MMP-2, MMP-9 and MMP-13), membrane-type matrix metalloproteinases and cathepsins, which degrade the ECM, are also involved in increased cancer cell invasion [5,6,56-58]. Pro-invasion soluble factors secreted from non-cancerous cells such as endothelial cells and immune cells in the tumor microenvironment [25,56,59-61] have also been reported to be involved in the invasive behavior of GSCs.

In conclusion, both 2D transwell invasion assays and 3D spheroid invasion assays are accurate and reproducible in vitro methodologies to study GSC invasion and are also applicable to other types of cancer. We conclude that the $2 \mathrm{D}$ transwell invasion assay is faster and less complex and is therefore more suitable for the initial experimental determination of specific chemoattractant receptormediated cellular invasion, which can then be studied using the more time consuming and complex 3D spheroid invasion assay that is physiologically closer to the in vivo situation than the $2 \mathrm{D}$ transwell invasion assay.

\section{Future perspective}

The described in vitro invasion assays are excellent experimental tools for translational research to increase our understanding of glioblastoma biology and to facilitate the development of novel therapies that inhibit invasion of GSCs. The methodological principles are not exclusive for glioblastoma and can also be applied to other types of cancer (stem) cells. In addition, novel therapies designed on the basis of these methodologies can work synergistically with current therapeutic approaches such as chemotherapy, radiotherapy and/or immunotherapy.

\section{Author contributions}

Study conception: V Hira, B Breznik, R Molenaar. Experiments: V Hira, B Breznik. Analysis: V Hira, B Breznik. Manuscript drafting: V Hira, B Breznik, R Molenaar. Manuscript editing: all authors. Supervision: C Van Noorden, T Lah, R Molenaar. Funding: T Lah, R Molenaar. Final approval of the manuscript: all authors.

\section{Financial \& competing interests disclosure}

This study was financially supported by the Dutch Cancer Society (KWF; UVA 2014-6839 and UVA 2016-10460). V Hira was supported by the IVY Interreg Fellowship 2018, R Molenaar was supported by the Fondation pour la Recherche Nuovo-Soldati 2019, T Lah was supported by the Slovenian Research Agency (Program P10245) and the European Program of Cross-Border Cooperation for SloveniaItaly Interreg TRANS-GLIOMA (Program 2017) and B Breznik was supported by postdoctoral grant of Slovenian Research Agency (Project Z3-1870). The authors have no other relevant affiliations or financial involvement with any organization or entity with a financial interest in or financial conflict with the subject matter or materials discussed in the manuscript apart from those disclosed.

No writing assistance was utilized in the production of this manuscript.

\section{Ethical conduct of research}

The study was approved by the National Medical Ethics Committee of the Republic of Slovenia (approval no. 0120-190/2018/4).

\section{Open access}

This work is licensed under the Attribution-NonCommercial-NoDerivatives 4.0 Unported License. To view a copy of this license, visit http://creativecommons.org/licenses/by-nc-nd/4.0/

\section{References}

1. Galarza S, Kim H, Atay N, Peyton SR, Munson JM. 2D or 3D? How cell motility measurements are conserved across dimensions in vitro and translate in vivo. Bioeng. Transl. Med. 5(1), e10148 (2020).

2. Carmeliet P, Jain RK. Angiogenesis in cancer and other diseases. Nature 407(6801), 249-257 (2000).

3. de Gooijer MC, Guillen Navarro M, Bernards R, Wurdinger T, van Tellingen O. An experimenter's guide to glioblastoma invasion pathways. Trends Mol. Med. 24(9), 763-780 (2018).

4. Lefranc F, Brotchi J, Kiss R. Possible future issues in the treatment of glioblastomas: special emphasis on cell migration and the resistance of migrating glioblastoma cells to apoptosis. J. Clin. Oncol. 23(10), 2411-2422 (2005).

5. Gole B, Huszthy PC, Popovic M et al. The regulation of cysteine cathepsins and cystatins in human gliomas. Int. J. Cancer 131(8), 1779-1789 (2012).

6. Lah TT, Duran Alonso MB, Van Noorden CJ. Antiprotease therapy in cancer: hot or not? Expert Opin. Biol. Ther. 6(3), 257-279 (2006).

7. Bellail AC, Hunter SB, Brat DJ, Tan C, Van Meir EG. Microregional extracellular matrix heterogeneity in brain modulates glioma cell invasion. Int. J. Biochem. Cell Biol. 36(6), 1046-1069 (2004).

8. Rao SS, Lannutti JJ, Viapiano MS, Sarkar A, Winter JO. Toward 3D biomimetic models to understand the behavior of glioblastoma multiforme cells. Tissue Eng. Part B Rev. 20(4), 314-327 (2014).

9. Friedl P, Sahai E, Weiss S, Yamada KM. New dimensions in cell migration. Nat. Rev. Mol. Cell Biol. 13(11), 743-747 (2012).

10. Heffernan JM, Overstreet DJ, Le LD, Vernon BL, Sirianni RW. Bioengineered scaffolds for 3D analysis of glioblastoma proliferation and invasion. Ann. Biomed. Eng. 43(8), 1965-1977 (2015).

11. Sart S, Tomasi RF, Amselem G, Baroud CN. Multiscale cytometry and regulation of 3D cell cultures on a chip. Nat. Commun. 8(1), 469 (2017).

12. Hira VV, Verbovsek U, Breznik B et al. Cathepsin K cleavage of SDF-1alpha inhibits its chemotactic activity towards glioblastoma stem-like cells. Biochim. Biophys. Acta 1864(3), 594-603 (2017).

13. Gont A, Daneshmand M, Woulfe J, Lavictoire SJ, Lorimer IA. PREX1 integrates G protein-coupled receptor and phosphoinositide 3-kinase signaling to promote glioblastoma invasion. Oncotarget 8(5), 8559-8573 (2017).

14. Breznik B, Motaln H, Vittori M, Rotter A, Lah Turnsek T. Mesenchymal stem cells differentially affect the invasion of distinct glioblastoma cell lines. Oncotarget 8(15), 25482-25499 (2017). 
15. Moriconi C, Palmieri V, Di Santo R et al. INSIDIA: a FIJI macro delivering high-throughput and high-content spheroid invasion analysis. Biotechnol. J. 12(10), (2017).

16. Corcoran A, De Ridder LI, Del Duca D et al. Evolution of the brain tumour spheroid model: transcending current model limitations. Acta Neurochir. 145(9), 819-824 (2003).

17. Decaestecker C, Debeir O, Van Ham P, Kiss R. Can anti-migratory drugs be screened in vitro? A review of $2 \mathrm{D}$ and $3 \mathrm{D}$ assays for the quantitative analysis of cell migration. Med. Res. Rev. 27(2), 149-176 (2007).

18. Benjamin DC, Hynes RO. Intravital imaging of metastasis in adult zebrafish. BMC Cancer 17(1), 660 (2017).

19. Hirata E, Girotti MR, Viros A et al. Intravital imaging reveals how BRAF inhibition generates drug-tolerant microenvironments with high integrin beta1/FAK signaling. Cancer Cell 27(4), 574-588 (2015).

20. Kallenberg K, Goldmann T, Menke J et al. Glioma infiltration of the corpus callosum: early signs detected by DTI. J. Neurooncol. 112(2), 217-222 (2013).

21. Cheng L, Wu Q, Guryanova OA et al. Elevated invasive potential of glioblastoma stem cells. Biochem. Biophys. Res. Commun. 406(4), 643-648 (2011).

22. Molina JR, Hayashi Y, Stephens C, Georgescu MM. Invasive glioblastoma cells acquire stemness and increased Akt activation. Neoplasia 12(6), 453-463 (2010).

23. Hira VV, Ploegmakers KJ, Grevers F et al. CD133+ and nestin+ glioma stem-like cells reside around CD31+ arterioles in niches that express SDF-1alpha, CXCR4, osteopontin and Cathepsin K. J. Histochem. Cytochem. 63(7), 481-493 (2015).

24. Hira VVV, Breznik B, Vittori M et al. Similarities between stem cell niches in glioblastoma and bone marrow: rays of hope for novel treatment strategies. J. Histochem. Cytochem. 68(1), 33-57 (2020).

25. Aderetti DA, Hira VVV, Molenaar RJ, van Noorden CJF. The hypoxic peri-arteriolar glioma stem cell niche, an integrated concept of five types of niches in human glioblastoma. Biochim. Biophys. Acta Rev. Cancer 1869(2), 346-354 (2018).

26. Zagzag D, Lukyanov Y, Lan L et al. Hypoxia-inducible factor 1 and VEGF upregulate CXCR4 in glioblastoma: implications for angiogenesis and glioma cell invasion. Lab. Invest. 86(12), 1221-1232 (2006).

27. Ehtesham M, Winston JA, Kabos P, Thompson RC. CXCR4 expression mediates glioma cell invasiveness. Oncogene 25(19), 2801-2806 (2006).

28. Monteiro AR, Hill R, Pilkington GJ, Madureira PA. The role of hypoxia in glioblastoma invasion. Cells 6(4), 45 (2017).

29. Hong X, Jiang F, Kalkanis SN et al. SDF-1 and CXCR4 are up-regulated by VEGF and contribute to glioma cell invasion. Cancer Lett. 236(1), 39-45 (2006).

30. Podergajs N, Motaln H, Rajcevic U et al. Transmembrane protein CD9 is glioblastoma biomarker, relevant for maintenance of glioblastoma stem cells. Oncotarget 7(1), 593-609 (2016).

31. Kolosa K, Motaln H, Herold-Mende C, Korsic M, Lah TT. Paracrine effects of mesenchymal stem cells induce senescence and differentiation of glioblastoma stem-like cells. Cell Transplant. 24(4), 631-644 (2015).

32. Schneider CA, Rasband WS, Eliceiri KW. NIH image to ImageJ: 25 years of image analysis. Nat. Methods 9(7), 671-675 (2012).

33. Chieco P, Jonker A, De Boer BA, Ruijter JM, Van Noorden CJ. Image cytometry: protocols for 2D and 3D quantification in microscopic images. Prog. Histochem. Cytochem. 47(4), 211-333 (2013).

34. Horing E, Harter PN, Seznec J et al. The 'go or grow' potential of gliomas is linked to the neuropeptide processing enzyme carboxypeptidase E and mediated by metabolic stress. Acta Neuropathol. 124(1), 83-97 (2012).

35. Hatzikirou H, Basanta D, Simon M, Schaller K, Deutsch A. 'Go or grow': the key to the emergence of invasion in tumour progression? Math. Med. Biol. 29(1), 49-65 (2012).

36. Bahmad HF, Chamaa F, Assi S, Chalhoub RM, Abou-Antoun T, Abou-Kheir W. Cancer stem cells in neuroblastoma: expanding the therapeutic frontier. Front. Mol. Neurosci. 12, 131 (2019).

37. Bahmad HF, Cheaito K, Chalhoub RM et al. Sphere-formation assay: three-dimensional in vitro culturing of prostate cancer stem/progenitor sphere-forming cells. Front. Oncol. 8 , 347 (2018).

38. Bahmad HF, Elajami MK, El Zarif T, Bou-Gharios J, Abou-Antoun T, Abou-Kheir W. Drug repurposing towards targeting cancer stem cells in pediatric brain tumors. Cancer Metastasis Rev. 39(1), 127-148 (2020).

39. Bahmad HF, Mouhieddine TH, Chalhoub RM et al. The Akt/mTOR pathway in cancer stem/progenitor cells is a potential therapeutic target for glioblastoma and neuroblastoma. Oncotarget 9(71), 33549-33561 (2018).

40. Bahmad HF, Poppiti RJ. Medulloblastoma cancer stem cells: molecular signatures and therapeutic targets. J. Clin. Pathol. 73(5), 243-249 (2020).

41. Mouhieddine TH, Nokkari A, Itani MM et al. Metformin and Ara-a effectively suppress brain cancer by targeting cancer stem/progenitor cells. Front. Neurosci. 9, 442 (2015).

42. Gole B, Duran Alonso MB, Dolenc V, Lah T. Post-translational regulation of cathepsin B, but not of other cysteine cathepsins, contributes to increased glioblastoma cell invasiveness in vitro. Pathol. Oncol. Res. 15(4), 711-723 (2009).

43. Motaln H, Turnsek TL. Cytokines play a key role in communication between mesenchymal stem cells and brain cancer cells. Protein Pept. Lett. 22(4), 322-331 (2015).

44. Hira VVV, Van Noorden CJF, Molenaar RJ. CXCR4 antagonists as stem cell mobilizers and therapy sensitizers for acute myeloid leukemia and glioblastoma? Biology (Basel) 9 (2), 31 (2020).

45. Chonan Y, Taki S, Sampetrean O, Saya H, Sudo R. Endothelium-induced three-dimensional invasion of heterogeneous glioma initiating cells in a microfluidic coculture platform. Integr. Biol. 9(9), 762-773 (2017)

46. Fernandes JT, Chutna O, Chu V, Conde JP, Outeiro TF. A novel microfluidic cell co-culture platform for the study of the molecular mechanisms of Parkinson's disease and other synucleinopathies. Front. Neurosci. 10, 511 (2016).

47. Gao Y, Majumdar D, Jovanovic B et al. A versatile valve-enabled microfluidic cell co-culture platform and demonstration of its applications to neurobiology and cancer biology. Biomed. Microdevices 13(3), 539-548 (2011).

48. Jacob F, Salinas RD, Zhang DY et al. A patient-derived glioblastoma organoid model and biobank recapitulates inter- and intra-tumoral heterogeneity. Cell 180(1), 188-204.e22 (2020).

49. Clevers H. Modeling development and disease with organoids. Cell 165(7), 1586-1597 (2016).

50. Bian S, Repic M, Guo Z et al. Genetically engineered cerebral organoids model brain tumor formation. Nat. Methods 15(8), 631-639 (2018).

51. Ogawa J, Pao GM, Shokhirev MN, Verma IM. Glioblastoma model using human cerebral organoids. Cell Rep. 23(4), 1220-1229 (2018).

52. da Silva B, Mathew RK, Polson ES, Williams J, Wurdak H. Spontaneous glioblastoma spheroid infiltration of early-stage cerebral organoids models brain tumor invasion. SLAS Discov. 23(8), 862-868 (2018)

53. Linkous A, Balamatsias D, Snuderl M et al. Modeling patient-derived glioblastoma with cerebral organoids. Cell Rep. 26(12), 3203-3211.e5 (2019).

54. Hubert CG, Rivera M, Spangler LC et al. A three-dimensional organoid culture system derived from human glioblastomas recapitulates the hypoxic gradients and cancer stem cell heterogeneity of tumors found in vivo. Cancer Res. 76(8), 2465-2477 (2016).

55. Chen HI, Song H, Ming GL. Applications of human brain organoids to clinical problems. Dev. Dyn. 248(1), 53-64 (2019).

56. Roos A, Ding Z, Loftus JC, Tran NL. Molecular and microenvironmental determinants of glioma stem-like cell survival and invasion. Front. Oncol. 7, 120 (2017).

57. Inoue A, Takahashi H, Harada H et al. Cancer stem-like cells of glioblastoma characteristically express MMP-13 and display highly invasive activity. Int. J. Oncol. 37(5), 1121-1131 (2010).

58. Mook OR, Frederiks WM, Van Noorden CJ. The role of gelatinases in colorectal cancer progression and metastasis. Biochim. Biophys. Acta 1705(2), 69-89 (2004).

59. Vollmann-Zwerenz A, Leidgens V, Feliciello G, Klein CA, Hau P. Tumor cell invasion in glioblastoma. Int. J. Mol. Sci. 21(6), 1932 (2020).

60. Xia S, Lal B, Tung B, Wang S, Goodwin CR, Laterra J. Tumor microenvironment tenascin-C promotes glioblastoma invasion and negatively regulates tumor proliferation. Neuro Oncol. 18(4), 507-517 (2016).

61. Wurth R, Bajetto A, Harrison JK, Barbieri F, Florio T. CXCL12 modulation of CXCR4 and CXCR7 activity in human glioblastoma stem-like cells and regulation of the tumor microenvironment Front. Cell. Neurosci. 8, 144 (2014). 